\title{
On the homotopy category of Moore spaces and the cohomology of the category of abelian groups
}

\author{
by
}

Hans-Joachim Ba ues (Bonn) and Manfred Hart l (Valenciennes)

\begin{abstract}
The homotopy category of Moore spaces in degree 2 represents a nontrivial cohomology class in the cohomology of the category of abelian groups. We describe various properties of this class. We use James-Hopf invariants to obtain explicitly the image category under the functor chain complex of the loop space.
\end{abstract}

An abelian group $A$ determines the Moore space $M(A)=M(A, 2)$ which up to homotopy equivalence is the unique simply connected $\mathrm{CW}$-space $X$ with homology groups $H_{2} X=A$ and $H_{i} X=0$ for $i>2$. Since $M(A)$ can be chosen to be a suspension, the set of homotopy classes $[M(A), M(B)]$ is a group which is part of a classical central extension of groups

$$
\operatorname{Ext}(A, \Gamma B) \longmapsto[M(A), M(B)] \rightarrow \operatorname{Hom}(A, B)
$$

due to Barratt. It is known that (1) in general is not split, for example $[M(\mathbb{Z} / 2), M(\mathbb{Z} / 2)]=\mathbb{Z} / 4$. We are not interested here in this additive structure of the sets $[M(A), M(B)]$ but in the multiplicative structure given by the composition of maps, in particular in the extension of groups

$$
\operatorname{Ext}(A, \Gamma A) \longmapsto \mathcal{E}(M(A)) \rightarrow \operatorname{Aut}(A),
$$

where $\mathcal{E}(M(A))$ is the group of homotopy equivalences of the space $M(A)$. The extension (2) determines the cohomology class

$$
\{\mathcal{E}(M(A))\} \in H^{2}(\operatorname{Aut}(A), \operatorname{Ext}(A, \Gamma A)) .
$$

Though the group $\mathcal{E}(M(A))$ is defined in an "easy" range of homotopy theory the cohomology class (3) is not yet computed for all abelian groups $A$.

In this paper we prove a nice algebraic formula for the class (3) if $A$ is a product of cyclic groups and we show that $\{\mathcal{E}(M(A))\}$ is trivial if

1991 Mathematics Subject Classification: 55E05, 55E25, 55J. 
$\operatorname{Ext}(A, \Gamma A)$ has no 2-torsion; see (3.6) and (5.2). Moreover, we compute for all abelian groups $A$ the image of the class (3) under the surjection of coefficients

$$
\operatorname{Ext}(A, \Gamma A) \rightarrow \operatorname{Ext}(A, H(\Gamma A)) .
$$

Here $H(\Gamma A)$ is the image of $H: \Gamma A \rightarrow A \otimes A$; see (4.2). We do such computations not in the cohomology of groups but more distinctly in the cohomology of categories. In fact, the homotopy category $\mathbf{M}^{2}$ of Moore spaces $M(A)$ leads to a topological "characteristic class" in the cohomology of the category $\mathbf{A} \mathbf{b}$ of abelian groups; see (2.2). It is the computation of such topologically defined cohomology classes which motivated the results in this paper. For example the topological James-Hopf invariant on the category $\mathbf{M}^{2}$ or the "chains on the loop space" functor $C_{*} \Omega$ on $\mathbf{M}^{2}$ have interesting interpretations on the level of the cohomology of the category $\mathbf{A b}$; see (4.11). As an application we describe algebraically the image category $\left(C_{*} \Omega\right)\left(\mathbf{M}^{2}\right)$ in the homotopy category of chain algebras showing fundamental differences between the homotopy category of spaces and chain algebras respectively; see (4.12). This implies that the image of the group $\mathcal{E}(M(A))$ under the functor $C_{*} \Omega$ is part of an extension

$$
\operatorname{Ext}(A, H(\Gamma A)) \longmapsto\left(C_{*} \Omega\right) \mathcal{E}(M(A)) \rightarrow \operatorname{Aut}(A),
$$

which we compute explicitly in terms of $A$ for all abelian groups $A$.

1. Linear extensions and cohomology of categories. An extension of a group $G$ by a $G$-module $A$ is a short exact sequence of groups

$$
0 \rightarrow A \underset{i}{\rightarrow} \underset{p}{\rightarrow} G \rightarrow 0,
$$

where $i$ is compatible with the action of $G$. Two such extensions $E$ and $E^{\prime}$ are equivalent if there is an isomorphism $\varepsilon: E \cong E^{\prime}$ of groups with $p^{\prime} \varepsilon=p$ and $\varepsilon i=i^{\prime}$. It is well known that the equivalence classes of extensions are classified by the cohomology $H^{2}(G, A)$.

We now recall from [2] the basic notation of the cohomology of categories. We describe linear extensions of a small category $\mathbf{C}$ by a "natural system" $D$. The equivalence classes of such extensions are classified by the cohomology $H^{2}(\mathbf{C}, D)$. A natural system $D$ on a category $\mathbf{C}$ is the appropriate generalization of a $G$-module.

(1.1) Definition. Let $\mathbf{C}$ be a category. The category of factorizations in $\mathbf{C}$, denoted by $F \mathbf{C}$, is given as follows. Objects are morphisms $f, g, \ldots$ in $\mathbf{C}$ and morphisms $f \rightarrow g$ are pairs $(\alpha, \beta)$ for which 


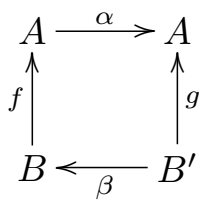

commutes in $\mathbf{C}$. Here $\alpha f \beta$ is a factorization of $g$. Composition is defined by $\left(\alpha^{\prime}, \beta^{\prime}\right)(\alpha, \beta)=\left(\alpha^{\prime} \alpha, \beta \beta^{\prime}\right)$. We clearly have $(\alpha, \beta)=(\alpha, 1)(1, \beta)=$ $(1, \beta)(\alpha, 1)$. A natural system (of abelian groups) on $\mathbf{C}$ is a functor $D$ : $F \mathbf{C} \rightarrow \mathbf{A b}$. The functor $D$ carries the object $f$ to $D_{f}=D(f)$ and carries the morphism $(\alpha, \beta): f \rightarrow g$ to the induced homomorphism

$$
D(\alpha, \beta)=\alpha_{*} \beta^{*}: D_{f} \rightarrow D_{\alpha f \beta}=D_{g} .
$$

Here we set $D(\alpha, 1)=\alpha_{*}, D(1, \beta)=\beta^{*}$.

We have a canonical forgetful functor $\pi: F \mathbf{C} \rightarrow \mathbf{C}^{\mathrm{op}} \times \mathbf{C}$ so that each bifunctor $D: \mathbf{C}^{\mathrm{op}} \times \mathbf{C} \rightarrow \mathbf{A b}$ yields a natural system $D \pi$, also denoted by $D$. Such a bifunctor is also called a C-bimodule. In this case $D_{f}=D(B, A)$ depends only on the objects $A, B$ for all $f \in \mathbf{C}(B, A)$. Two functors $F, G$ : $\mathbf{A b} \rightarrow \mathbf{A b}$ yield the $\mathbf{A b}$-bimodule

$$
\operatorname{Hom}(F, G): \mathbf{A} \mathbf{b}^{\text {op }} \times \mathbf{A} \mathbf{b} \rightarrow \mathbf{A b}
$$

which carries $(A, B)$ to the group of homomorphisms $\operatorname{Hom}(F A, G B)$. If $F$ is the identity functor we write $\operatorname{Hom}(-, G)$. Similarly we define the $\mathbf{A b}$ bimodule $\operatorname{Ext}(F, G)$.

For a group $G$ and a $G$-module $A$ the corresponding natural system $D$ on the group $G$, considered as a category, is given by $D_{g}=A$ for $g \in G$ and $g_{*} a=g \cdot a$ for $a \in A, g^{*} a=a$. If we restrict the following notion of a "linear extension" to the case $\mathbf{C}=G$ and $D=A$ we obtain the notion of a group extension above.

(1.2) Definition. Let $D$ be a natural system on $\mathbf{C}$. We say that

$$
D \stackrel{+}{\longmapsto} \mathbf{E} \stackrel{p}{\rightarrow} \mathbf{C}
$$

is a linear extension of the category $\mathbf{C}$ by $D$ if (a), (b) and (c) below hold.

(a) $\mathbf{E}$ and $\mathbf{C}$ have the same objects and $p$ is a full functor which is the identity on objects.

(b) For each $f: A \rightarrow B$ in $\mathbf{C}$, the abelian group $D_{f}$ acts transitively and effectively on the subset $p^{-1}(f)$ of morphisms in $\mathbf{E}$. We write $f_{0}+\alpha$ for the action of $\alpha \in D_{f}$ on $f_{0} \in p^{-1}(f)$.

(c) The action satisfies the linear distributivity law:

$$
\left(f_{0}+\alpha\right)\left(g_{0}+\beta\right)=f_{0} g_{0}+f_{*} \beta+g^{*} \alpha .
$$

Two linear extensions $\mathbf{E}$ and $\mathbf{E}^{\prime}$ are equivalent if there is an isomorphism of categories $\varepsilon: \mathbf{E} \cong \mathbf{E}^{\prime}$ with $p^{\prime} \varepsilon=p$ and with $\varepsilon\left(f_{0}+\alpha\right)=\varepsilon\left(f_{0}\right)+\alpha$ 
for $f_{0} \in \operatorname{Mor}(\mathbf{E}), \alpha \in D_{p f_{0}}$. The extension $\mathbf{E}$ is split if there is a functor $s: \mathbf{C} \rightarrow \mathbf{E}$ with $p s=1$. Let $M(\mathbf{C}, D)$ be the set of equivalence classes of linear extensions of $\mathbf{C}$ by $\mathbf{D}$. Then there is a canonical bijection

$$
\psi: M(\mathbf{C}, D) \cong H^{2}(\mathbf{C}, D)
$$

which maps the split extension to the zero element (see [2] and IV, $\S 6$ in [4]). Here $H^{n}(\mathbf{C}, D)$ denotes the cohomology of $\mathbf{C}$ with coefficients in $D$ which is defined below. We obtain a representing cocycle $\Delta_{t}$ of the cohomology class $\{\mathbf{E}\}=\psi(\mathbf{E}) \in H^{2}(\mathbf{C}, D)$ as follows. Let $t$ be a "splitting" function for $p$ which associates with each morphism $f: A \rightarrow B$ in $\mathbf{C}$ a morphism $f_{0}=t(f)$ in $\mathbf{E}$ with $p f_{0}=f$. Then $t$ yields a cocycle $\Delta_{t}$ by the formula

$$
t(g f)=t(g) t(f)+\Delta_{t}(g, f)
$$

with $\Delta_{t}(g, f) \in D(g f)$. The cohomology class $\{\mathbf{E}\}=\left\{\Delta_{t}\right\}$ is trivial if and only if $\mathbf{E}$ is a split extension.

(1.5) Definition. Let $\mathbf{C}$ be a small category and let $N_{n}(\mathbf{C})$ be the set of sequences $\left(\lambda_{1}, \ldots, \lambda_{n}\right)$ of $n$ composable morphisms in $\mathbf{C}$ (which are the $n$-simplices of the nerve of $\mathbf{C}$ ). For $n=0$ let $N_{0}(\mathbf{C})=\mathrm{Ob}(\mathbf{C})$ be the set of objects in $\mathbf{C}$. The cochain group $F^{n}=F^{n}(\mathbf{C}, D)$ is the abelian group of all functions

$$
c: N_{n}(\mathbf{C}) \rightarrow\left(\bigcup_{g \in \operatorname{Mor}(\mathbf{C})} D_{g}\right)=D
$$

with $c\left(\lambda_{1}, \ldots, \lambda_{n}\right) \in D_{\lambda_{1} \circ \ldots \circ \lambda_{n}}$. Addition in $F^{n}$ is given by adding pointwise in the abelian groups $D_{g}$. The coboundary $\partial: F^{n-1} \rightarrow F^{n}$ is defined by the formula

$$
\begin{aligned}
(\partial c)\left(\lambda_{1}, \ldots, \lambda_{n}\right)= & \left(\lambda_{1}\right)_{*} c\left(\lambda_{2}, \ldots, \lambda_{n}\right) \\
& +\sum_{i=1}^{n-1}(-1)^{i} c\left(\lambda_{1}, \ldots, \lambda_{i} \lambda_{i+1}, \ldots, \lambda_{n}\right) \\
& +(-1)^{n}\left(\lambda_{n}\right)^{*} c\left(\lambda_{1}, \ldots, \lambda_{n-1}\right) .
\end{aligned}
$$

For $n=1$ we have $(\partial c)(\lambda)=\lambda_{*} c(A)-\lambda^{*} c(B)$ for $\lambda: A \rightarrow B \in N_{1}(\mathbf{C})$. One can check that $\partial c \in F^{n}$ for $c \in F^{n-1}$ and that $\partial \partial=0$. Hence the cohomology groups

$$
H^{n}(\mathbf{C}, D)=H^{n}\left(F^{*}(\mathbf{C}, D), \partial\right)
$$

are defined for $n \geq 0$. These groups are discussed in [9] and [2]. By change of the universe cohomology groups $H^{n}(\mathbf{C}, D)$ can also be defined if $\mathbf{C}$ is not a small category. A functor $\phi: \mathbf{C}^{\prime} \rightarrow \mathbf{C}$ induces the homomorphism

$$
\phi^{*}: H^{n}(\mathbf{C}, D) \rightarrow H^{n}\left(\mathbf{C}^{\prime}, \phi^{*} D\right),
$$


where $\phi^{*} D$ is the natural system given by $\left(\phi^{*} D\right)_{f}=D_{\phi(f)}$. On cochains the map $\phi^{*}$ is given by the formula

$$
\left(\phi^{*} f\right)\left(\lambda_{1}^{\prime}, \ldots, \lambda_{n}^{\prime}\right)=f\left(\phi \lambda_{1}^{\prime}, \ldots, \phi \lambda_{n}^{\prime}\right),
$$

where $\left(\lambda_{1}^{\prime}, \ldots, \lambda_{n}^{\prime}\right) \in N_{n}\left(\mathbf{C}^{\prime}\right)$. If $\phi$ is an equivalence of categories then $\phi^{*}$ is an isomorphism. A natural transformation $\tau: D \rightarrow D^{\prime}$ between natural systems induces a homomorphism

$$
\tau_{*}: H^{n}(\mathbf{C}, D) \rightarrow H^{n}\left(\mathbf{C}, D^{\prime}\right)
$$

by $\left(\tau_{*} f\right)\left(\lambda_{1}, \ldots, \lambda_{n}\right)=\tau_{\lambda} f\left(\lambda_{1}, \ldots, \lambda_{n}\right)$ where $\tau_{\lambda}: D_{\lambda} \rightarrow D_{\lambda}^{\prime}$ with $\lambda=$ $\lambda_{1} \circ \ldots \circ \lambda_{n}$ is given by the transformation $\tau$. Now let

$$
D^{\prime \prime} \stackrel{l}{\longmapsto} D \stackrel{\tau}{\rightarrow} D^{\prime}
$$

be a short exact sequence of natural systems on $\mathbf{C}$. Then we obtain as usual the natural long exact sequence

$$
\rightarrow H^{n}\left(\mathbf{C}, D^{\prime}\right) \stackrel{l_{*}}{\rightarrow} H^{n}(\mathbf{C}, D) \stackrel{\tau_{*}}{\rightarrow} H^{n}\left(\mathbf{C}, D^{\prime \prime}\right) \stackrel{\beta}{\rightarrow} H^{n+1}\left(\mathbf{C}, D^{\prime}\right) \rightarrow,
$$

where $\beta$ is the Bockstein homomorphism. For a cocycle $c^{\prime \prime}$ representing a class $\left\{c^{\prime \prime}\right\}$ in $H^{n}\left(\mathbf{C}, D^{\prime \prime}\right)$ we obtain $\beta\left\{c^{\prime \prime}\right\}$ by choosing a cochain $c$ as in (1.5)(1) with $\tau c=c^{\prime \prime}$. This is possible since $\tau$ is surjective. Then $\iota^{-1} \delta c$ is a cocycle which represents $\beta\left\{c^{\prime \prime}\right\}$.

(1.7) Remark. The cohomology (1.5) generalizes the cohomology of a group. In fact, let $G$ be a group and let $\mathbf{G}$ be the corresponding category with a single object and with morphisms given by the elements in $G$. A $G$-module $A$ yields a natural system $D$. Then the classical definition of the cohomology $H^{n}(G, A)$ coincides with the definition of

$$
H^{n}(\mathbf{G}, D)=H^{n}(G, A)
$$

given by (1.5). Further results and applications of the cohomology of categories can be found in [2], [3], [8], [9], [13], [14].

2. The homotopy category $\mathbf{M}^{2}$ of Moore spaces in degree 2. Let $A$ be an abelian group. A Moore space $M(A, n), n \geq 2$, is a simply connected CW-space $X$ with (reduced) homology groups $H_{n} X=A$ and $H_{i} X=0$ for $i \neq n$. An Eilenberg-MacLane space $K(A, n)$ is a CW-space $Y$ with homotopy groups $\pi_{n} Y=A$ and $\pi_{i} Y=0$ for $i \neq n$. Such spaces exist and their homotopy type is well defined by $(A, n)$. The homotopy category of Eilenberg-MacLane spaces $K(A, n), A \in \mathbf{A} \mathbf{b}$, is isomorphic via the functor $\pi_{n}$ to the category $\mathbf{A b}$ of abelian groups. The corresponding result, however, does not hold for the homotopy category $\mathbf{M}^{n}$ of Moore spaces $M(A, n)$, $A \in \mathbf{A b}$. This creates the problem to find a suitable algebraic model of the category $\mathbf{M}^{n}$. For $n \geq 3$ such a model category of $\mathbf{M}^{n}$ is known (see (V.3a.8) in [2] and $(\mathrm{I}, \S 6)$ in [4]). The category $\mathbf{M}^{2}$ is not completely understood. We 
shall use the cohomology of the category $\mathbf{A b}$ to describe various properties of the category $\mathbf{M}^{2}$.

Let $\Gamma: \mathbf{A b} \rightarrow \mathbf{A b}$ be J. H. C. Whitehead's quadratic functor [15] with

$$
\Gamma(A)=\pi_{3} M(A, 2)=H_{4} K(A, 2) .
$$

Then we obtain the Ab-bimodule

$$
\operatorname{Ext}(-, \Gamma): \mathbf{A b}^{\text {op }} \times \mathbf{A b} \rightarrow \mathbf{A b}
$$

which carries $(A, B)$ to the group $\operatorname{Ext}(A, \Gamma(B))$.

(2.2) Proposition. The category $\mathbf{M}^{2}$ is part of a nonsplit linear extension

$$
\operatorname{Ext}(-, \Gamma) \stackrel{+}{\longmapsto} \mathbf{M}^{2} \stackrel{H_{2}}{\rightarrow} \mathbf{A b}
$$

and hence $\mathbf{M}^{2}$, up to equivalence, is characterized by a cohomology class

$$
\left\{\mathbf{M}^{2}\right\} \in H^{2}(\mathbf{A b}, \operatorname{Ext}(-, \Gamma)) .
$$

Since the extension is nonsplit we have $\left\{\mathbf{M}^{2}\right\} \neq 0$.

Pr o of. For a free abelian group $A_{0}$ with basis $Z$ let

$$
M_{A_{0}}=\bigvee_{Z} S^{1}
$$

be a one-point union of 1-dimensional spheres $S^{1}$ such that $H_{1} M_{A_{0}}=A_{0}$. For an abelian group $A$ we choose a short exact sequence

$$
0 \rightarrow A_{1} \stackrel{d_{A}}{\rightarrow} A_{0} \rightarrow A \rightarrow 0,
$$

where $A_{0}, A_{1}$ are free abelian. Let $d_{A}^{\prime}: M_{A_{1}} \rightarrow M_{A_{0}}$ be a map which induces $d_{A}$ in homology and let $M_{A}$ be the mapping cone of $d_{A}^{\prime}$. Then

$$
M(A, 2)=\Sigma M_{A}
$$

is the suspension of $M_{A}$. The homotopy type of $M_{A}$, however, depends on the choice of $d_{A}^{\prime}$ and is not determined by $A$. Using the cofiber sequence for $d_{A}^{\prime}$ we obtain the well known exact sequence of groups [11]

$$
0 \rightarrow \operatorname{Ext}\left(A, \pi_{3} X\right) \stackrel{\Delta}{\rightarrow}[M(A, 2), X] \stackrel{\mu}{\rightarrow} \operatorname{Hom}\left(A, \pi_{2} X\right) \rightarrow 0,
$$

where $[Y, X]$ denotes the set of homotopy classes of pointed maps $Y \rightarrow X$. We now set $X=M(B, 2)$. Then $\mu$ is given by the homology functor. We define the action of $\alpha \in \operatorname{Ext}(A, \Gamma B)$ on $\xi \in[M(A, 2), M(B, 2)]$ by $\xi+\alpha=$ $\xi+\Delta(\alpha)$ where we use the group structure in $\left[\Sigma M_{A}, M(B, 2)\right]$. This action satisfies the linear distributivity law so that we obtain the linear extension in (2.2). Compare also $(\mathrm{V}, \S 3 \mathrm{a})$ in $[2]$ where we show $\left\{\mathbf{M}^{2}\right\} \neq 0$.

(2.3) Remark. A Pontryagin map $\tau_{A}$ for an abelian group $A$ is a map

$$
\tau_{A}: K(A, 2) \rightarrow K(\Gamma(A), 4)
$$


which induces the identity of $\Gamma(A)$,

$$
\Gamma(A)=H_{4} K(A, 2) \rightarrow H_{4} K(\Gamma(A), 4)=\Gamma(A) .
$$

Such Pontryagin maps exist and are well defined up to homotopy. The map $\tau_{A}$ induces the Pontryagin square which is the cohomology operation [15]

$$
H^{2}(X, A)=[X, K(A, 2)] \stackrel{\left(\tau_{A}\right)_{*}}{\longrightarrow}[X, K(\Gamma(A), 2)]=H^{4}(X, \Gamma(A)) .
$$

The fiber of $\tau_{A}$ is the 3 -type of $M(A, 2)$. Therefore one gets isomorphisms of categories [7]

$$
\mathbf{M}^{2}=\mathbf{P}(\mathcal{X})=\text { Hopair }(\mathcal{X}),
$$

where $\mathcal{X}$ is the class of all Pontryagin maps $\tau_{A}, A \in \mathbf{A b}$. Here $\mathbf{P}(\mathcal{X})$ is the homotopy category of fibers $P\left(\tau_{A}\right), \tau_{A} \in \mathcal{X}$, and Hopair $(\mathcal{X})$ is the category of homotopy pairs [10] between Pontryagin maps. We have seen in [9] that via these isomorphisms the class $\left\{\mathbf{M}^{2}\right\}$ is the image of the universal Toda bracket $\langle\mathbf{K}\rangle_{\Omega} \in H^{3}\left(\mathbf{K}, D_{\Omega}\right)$ where $\mathbf{K}$ is the full subcategory of the homotopy category consisting of $K(A, 2)$ and $K(\Gamma(A), 4), A \in \mathbf{A b}$. Hence we get by $(2.2)$ :

(2.4) Corollary. $\langle\mathbf{K}\rangle_{\Omega} \neq 0$.

3. On the cohomology class $\left\{\mathbf{M}^{2}\right\}$. The quadratic functor $\Gamma$ can also be defined by the universal quadratic map $\gamma: A \rightarrow \Gamma(A)$. We have the natural exact sequence in $\mathbf{A b}$

$$
\Gamma(A) \stackrel{H}{\rightarrow} A \otimes A \stackrel{q}{\rightarrow} \Lambda^{2} A \rightarrow 0,
$$

where $H$ is defined by $H \gamma(a)=a \otimes a, a \in A \in \mathbf{A b}$, and where $\Lambda^{2} A=$ $A \otimes A /\{a \otimes a \sim 0\}$ is the exterior square with quotient map $q$. We also need the natural homomorphism

$$
[1,1]=P: A \otimes A \rightarrow \Gamma(A)
$$

with $P(a \otimes b)=\gamma(a+b)-\gamma(a)-\gamma(b)=[a, b]$. One readily checks that $P H$ is multiplication by 2 on $\Gamma(A)$ and that $H P(a \otimes b)=a \otimes b+b \otimes a$. For $A \in \mathbf{A b}$ using $P$ and $H$ and $q$ above we obtain the following natural short exact sequences of $\mathbb{Z} / 2$-vector spaces:

$$
\begin{aligned}
& S_{1}(A): \Lambda^{2}(A) \otimes \mathbb{Z} / 2 \stackrel{P}{\longmapsto} \Gamma(A) \otimes \mathbb{Z} / 2 \stackrel{\sigma}{\rightarrow} A \otimes \mathbb{Z} / 2, \\
& S_{2}(A): \Gamma(A) \otimes \mathbb{Z} / 2 \stackrel{H}{\longmapsto} \otimes^{2}(A) \otimes \mathbb{Z} / 2 \stackrel{q}{\rightarrow} \Lambda^{2}(A) \otimes \mathbb{Z} / 2 .
\end{aligned}
$$

Here $\sigma$ carries $\gamma(a) \otimes 1$ to $a \otimes 1, a \in A$. If we apply the functor $\operatorname{Hom}(-, \Gamma(B) \otimes$ $\mathbb{Z} / 2)$ to the exact sequence $S_{i}(A), i=1,2$, we get the corresponding exact sequence of Ab-bimodules denoted by $\operatorname{Hom}\left(S_{i}(-), \Gamma(-) \otimes \mathbb{Z} / 2\right)$. The asso- 
ciated Bockstein homomorphisms $\beta_{i}$ yield thus homomorphisms

$$
\begin{aligned}
& H^{0}(\mathbf{A b}, \operatorname{Hom}(\Gamma(-) \otimes \mathbb{Z} / 2, \Gamma(-) \otimes \mathbb{Z} / 2)) \\
& \quad \downarrow \beta_{2} \\
& H^{1}\left(\mathbf{A b}, \operatorname{Hom}\left(\Lambda^{2}(-) \otimes \mathbb{Z} / 2, \Gamma(-) \otimes \mathbb{Z} / 2\right)\right) \\
& \quad \downarrow \beta_{1} \\
& H^{2}(\mathbf{A b}, \operatorname{Hom}(-\otimes \mathbb{Z} / 2, \Gamma(-) \otimes \mathbb{Z} / 2))
\end{aligned}
$$

Moreover, we use the natural homomorphism

$$
\chi: \operatorname{Hom}(A \otimes \mathbb{Z} / 2, \Gamma(B) \otimes \mathbb{Z} / 2) \stackrel{g}{=} \operatorname{Ext}(A \otimes \mathbb{Z} / 2, \Gamma B) \stackrel{p^{*}}{\rightarrow} \operatorname{Ext}(A, \Gamma B),
$$

where $g$ is the natural isomorphism and where $p: A \rightarrow A \otimes \mathbb{Z} / 2$ is the projection. Let

$$
1_{\Gamma} \in H^{0}(\mathbf{A b}, \operatorname{Hom}(\Gamma(-) \otimes \mathbb{Z} / 2, \Gamma(-) \otimes \mathbb{Z} / 2))
$$

be the canonical class which carries the abelian group $A$ to the identity of $\Gamma(A) \otimes \mathbb{Z} / 2$. Then one gets the element

$$
\chi_{*} \beta_{1} \beta_{2}\left(1_{\Gamma}\right) \in H^{2}(\mathbf{A b}, \operatorname{Ext}(-, \Gamma))
$$

determined by $1_{\Gamma}$ and the homomorphisms above.

(3.5) Conjecture. $\left\{\mathbf{M}^{2}\right\}=\chi_{*} \beta_{1} \beta_{2}\left(1_{\Gamma}\right)$.

We shall prove various results which support this conjecture.

(3.6) Theorem. Let $\mathbf{A}$ be the full subcategory of $\mathbf{A b}$ consisting of direct sums of cyclic groups and let $i_{\mathbf{A}}: \mathbf{A} \rightarrow \mathbf{A b}$ be the inclusion functor. Then we have

$$
i_{\mathbf{A}}^{*}\left\{\mathbf{M}^{2}\right\}=i_{\mathbf{A}}^{*} \chi_{*} \beta_{1} \beta_{2}\left(1_{\gamma}\right) \in H^{2}(\mathbf{A}, \operatorname{Ext}(-, \Gamma)) .
$$

Proof. We write $C=(\mathbb{Z} / a) \alpha=\alpha(\mathbb{Z} / a)$ if $C$ is a cyclic group isomorphic to $\mathbb{Z} / a$ with generator $\alpha, a \geq 0$. A direct sum of cyclic groups

$$
A=\bigoplus_{i}\left(\mathbb{Z} / a_{i}\right) \alpha_{i}
$$

is indexed by an ordered set if the set of generators $\left\{\alpha_{i},<\right\}$ is a well ordered set. The generator $\alpha_{i}$ also denotes the inclusion $\alpha_{i}: \mathbb{Z} / a_{i} \subset A$ and the corresponding inclusion

$$
\alpha_{i}: \Sigma P_{a_{i}} \subset \bigvee_{i} \Sigma P_{a_{i}}=M(A, 2) .
$$

Here $P_{n}=S^{1} \cup_{n} e^{2}$ is the pseudo-projective plane for $n>0$ and $P_{0}=S^{1}$ so that $\Sigma P_{n}=M(\mathbb{Z} / n, 2)$. Let $\alpha^{i}: A \rightarrow \mathbb{Z} / a_{i}$ be the canonical retraction of $\alpha_{i}$ with $\alpha^{i} \alpha_{i}=1$ and $\alpha^{j} \alpha_{i}=0$ for $j \neq i$. Let

$$
\varphi: A=\bigoplus_{i} \alpha_{i}\left(\mathbb{Z} / a_{i}\right) \rightarrow B=\bigoplus_{j} \beta_{j}\left(\mathbb{Z} / b_{j}\right)
$$


be a homomorphism. The coordinates $\varphi_{j i} \in \mathbb{Z}, \varphi_{j i}: \mathbb{Z} / a_{i} \rightarrow \mathbb{Z} / b_{j}, \mathbf{1} \mapsto$ $\varphi_{j i} \mathbf{1}$, are given by the formula

$$
\varphi \alpha_{i}=\sum \beta_{j} \varphi_{j i}
$$

Let $B_{2}$ be the splitting function

$$
\left[\Sigma P_{n}, \Sigma P_{m}\right] \underset{B_{2}}{\rightleftarrows} \operatorname{Hom}(\mathbb{Z} / n, \mathbb{Z} / m)
$$

obtained in (III, Appendix D) of [3]. We define the map $s \varphi \in[M(A, 2)$, $M(B, 2)]$ by the ordered sum

$$
(s \varphi) \alpha_{i}=\sum_{j}^{<} \beta_{j} B_{2}\left(\varphi_{j i}\right),
$$

where we use the ordering $<$ of the generators in $B$. Hence we obtain a splitting function $s$ :

$$
[M(A, 2), M(B, 2)] \underset{s}{\stackrel{H_{2}}{\rightleftarrows}} \operatorname{Hom}(A, B)
$$

with $H_{2} s(\varphi)=\varphi$. Each element $\bar{\varphi} \in[M(A, 2), M(B, 2)]$ is of the form $\bar{\varphi}=$ $s(\varphi)+\xi$, where $\xi \in \operatorname{Ext}(A, \Gamma B)$. This way we can characterize all elements in $[M(A, 2), M(B, 2)]$ provided $A$ and $B$ are ordered direct sums of cyclic groups. We use $s$ in (3.9) for the definition of the cocycle $\Delta_{s}$ representing $i^{*}\left\{\mathbf{M}^{2}\right\}$ in (3.6), that is, by (1.4),

$$
s(\psi \varphi)=s(\psi) s(\varphi)+\Delta_{s}(\psi, \varphi) .
$$

Below we compute $\Delta_{s}$. To this end we have to introduce the following groups.

(3.10) Definition. Let $A$ be an abelian group. We have the natural homomorphism between $\mathbb{Z} / 2$-vector spaces

$$
H: \Gamma(A) \otimes \mathbb{Z} / 2=\Gamma(A \otimes \mathbb{Z} / 2) \otimes \mathbb{Z} / 2 \rightarrow \otimes^{2}(A \otimes \mathbb{Z} / 2)
$$

with $H(\gamma(a) \otimes 1)=(a \otimes 1) \otimes(a \otimes 1)$. This homomorphism is injective and hence admits a retraction homomorphism

$$
r: \otimes^{2}(A \otimes \mathbb{Z} / 2) \rightarrow \Gamma(A) \otimes \mathbb{Z} / 2
$$

with $r H=$ id. For example, given a basis $E$ of the $\mathbb{Z} / 2$-vector space $A \otimes \mathbb{Z} / 2$ and a well ordering $<$ on $E$ we can define a retraction $r^{<}$on the basis elements by the formula $\left(b, b^{\prime} \in E\right)$

$$
r^{<}\left(b \otimes b^{\prime}\right)= \begin{cases}\gamma(b) \otimes 1 & \text { for } b=b^{\prime} \\ {\left[b, b^{\prime}\right] \otimes 1} & \text { for } b>b^{\prime} \\ 0 & \text { for } b<b^{\prime}\end{cases}
$$

Now let $q \geq 1$ and let

$$
j_{A}: \operatorname{Hom}(\mathbb{Z} / q, A)=A * \mathbb{Z} / q \subset A \stackrel{p}{\rightarrow} A \otimes \mathbb{Z} / 2
$$


be given by the projection $p$ with $p(x)=x \otimes 1$. Also let

$$
\begin{aligned}
p_{A}: \Gamma(A) \otimes \mathbb{Z} / 2 \stackrel{p}{\longmapsto} \Gamma(A) \otimes \mathbb{Z} / 2 \otimes \mathbb{Z} / q \\
\quad=\operatorname{Ext}(\mathbb{Z} / 2 \otimes \mathbb{Z} / q, \Gamma(A)) \stackrel{p^{*}}{\rightarrow} \operatorname{Ext}(\mathbb{Z} / q, \Gamma(A))
\end{aligned}
$$

be defined by the indicated projections $p$. Then we obtain the homomorphism

$$
\begin{aligned}
& \Delta_{A}: \operatorname{Hom}(\mathbb{Z} / q, A) \otimes \operatorname{Hom}(\mathbb{Z} / q, A) \rightarrow \operatorname{Ext}(\mathbb{Z} / q, \Gamma A), \\
& \Delta_{A}=p_{A} r\left(j_{A} \otimes j_{A}\right),
\end{aligned}
$$

which depends on the choice of the retraction $r$ in (2). Clearly $\Delta_{A}$ is not natural in $A$ since $r$ cannot be chosen to be natural. However, one can easily check that $\Delta_{A}$ is natural for homomorphisms $\varphi: \mathbb{Z} / q \rightarrow \mathbb{Z} / t$ between cyclic groups, that is,

$$
\Delta_{A}\left(\varphi^{*} \otimes \varphi^{*}\right)=\varphi^{*} \Delta_{A} .
$$

We now define a group

$$
G(q, A)=\operatorname{Hom}(\mathbb{Z} / q, A) \times \operatorname{Ext}(\mathbb{Z} / q, \Gamma(A)),
$$

where the group law on the right-hand side is given by the cocycle $\Delta_{A}$, that is,

$$
(a, b)+\left(a^{\prime}, b^{\prime}\right)=\left(a+a^{\prime}, b+b^{\prime}+\Delta_{A}\left(a \otimes a^{\prime}\right)\right) .
$$

For any abelian group $A$, by (XII.1.6) of [4] there is an isomorphism

$$
\varrho: G(q, A) \cong\left[\Sigma P_{q}, M(A, 2)\right]
$$

which is natural in $\mathbb{Z} / q, q>1$, and which is compatible with $\Delta$ and $\mu$ in the proof of (2.2). If $A$ is a direct sum of cyclic groups as above, we obtain maps

$$
\bar{\alpha}_{i}: \Sigma P_{a_{i}} \rightarrow M(A, 2)
$$

defined by $\bar{\alpha}_{i}=\varrho\left(\alpha_{i}, 0\right)$, where $\alpha_{i} \in \operatorname{Hom}\left(\mathbb{Z} / a_{i}, A\right)$ is the inclusion. These maps yield the homotopy equivalence

$$
\bigvee_{i} \Sigma P_{a_{i}} \simeq M(A, 2)
$$

which we use as an identification. Hence we may assume that $\varrho$ in (3.11) satisfies

$$
\varrho\left(\alpha_{i}, 0\right)=\alpha_{i},
$$

where $\alpha_{i}$ is the inclusion in (3.7). We need the following function $\nabla_{A}$, defined 
for an ordered direct sum $A$ of cyclic groups,

$$
\begin{aligned}
& \nabla_{A}: \operatorname{Hom}(\mathbb{Z} / q, A) \rightarrow \operatorname{Ext}(\mathbb{Z} / q, \Gamma A), \\
& \nabla_{A}(x)=\sum_{i<j} \Delta_{A}\left(\alpha_{i} x_{i} \otimes \alpha_{j} x_{j}\right) .
\end{aligned}
$$

Here $x_{i} \in \operatorname{Hom}\left(\mathbb{Z} / q, \mathbb{Z} / a_{i}\right)$ is the coordinate of $x=\sum_{i} \alpha_{i} x_{i}$. We observe that $\nabla_{A}=0$ is trivial if we define $\Delta_{A}$ by $r^{<}$in (3.10), where the ordered basis $E$ in $A \otimes \mathbb{Z} / 2$ is given by the ordered set of generators in $A$. Clearly $2 \nabla_{A}(x)=0$ since $2 \Delta_{A}=0$. The function $\nabla_{A}$ has the following crucial property:

(3.13) Lemma. In the group $G(q, A)$ we have the formula

$$
\sum_{i}^{<} x_{i}^{*}\left(\alpha_{i}, 0\right)=\left(x, \nabla_{A}(x)\right),
$$

where the left-hand side is the ordered sum of the elements $x_{i}^{*}\left(\alpha_{i}, 0\right)=$ $\left(\alpha_{i} x_{i}, 0\right)$ in the group $G(q, A)$.

The lemma is an immediate consequence of the group law (3.10)(9).

For $\varphi \in \operatorname{Hom}(A, B)$ in (3.8) and $q \geq 1$ we define the function

$$
\nabla(\varphi): \operatorname{Hom}(\mathbb{Z} / q, A) \rightarrow \operatorname{Ext}(\mathbb{Z} / q, \Gamma(B))
$$

via the following commutative diagram, in which $\pi_{2}(\mathbb{Z} / q, M(A, 2))=$ $[M(\mathbb{Z} / q, 2), M(A, 2)]$ :

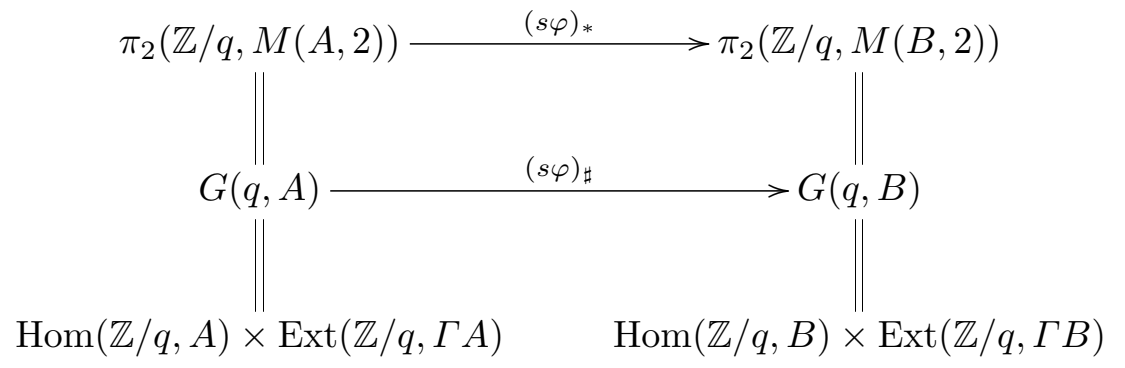

Here the isomorphisms are given as in (3.11). The homomorphism $(s \varphi)_{\sharp}$, induced by $s \varphi$ in (3.9), determines $\nabla(\varphi)$ by the formula

$$
(s \varphi)_{\sharp}(x, \alpha)=\left(\varphi_{*} x, \Gamma(\varphi)_{*} \alpha+\nabla(\varphi)(x)\right)
$$

for $x \in \operatorname{Hom}(\mathbb{Z} / q, A)$ and $\alpha \in \operatorname{Ext}(\mathbb{Z} / q, \Gamma A)$. The function $\nabla(\varphi)$ is not a homomorphism.

(3.15) Lemma. For $x \in \operatorname{Hom}(\mathbb{Z} / q, A)$ we have

$$
\nabla(\varphi)(x)=\Gamma(\varphi)_{*} \nabla_{A}(x)+\sum_{i} \nabla_{B}\left(\varphi \alpha_{i} x_{i}\right)+\sum_{i<t} \Delta_{B}\left(\varphi \alpha_{i} x_{i} \otimes \varphi \alpha_{t} x_{t}\right) .
$$

Since all summands are 2-torsion we have $\nabla(\varphi)=0$ if $q$ is odd. 
Proof. For $\left(\alpha_{i}, 0\right) \in G\left(a_{i}, A\right)$ one has the formula

$$
(s \varphi)_{\sharp}\left(\alpha_{i}, 0\right)=\sum_{j}^{<}\left(\beta_{j} \varphi_{j i}, 0\right),
$$

as follows from property $(3.11)(*)$ of the isomorphism $\chi$. Hence by (3.13) we get the following equations:

$$
\begin{aligned}
& (s \varphi)_{\sharp}(x, 0)+\left(0, \Gamma(\varphi)_{*} \nabla_{A}(x)\right) \\
& =(s \varphi)_{\sharp}\left(x, \nabla_{A}(x)\right)=(s \varphi)_{\sharp}\left(\sum_{i}^{<} x_{i}^{*}\left(\alpha_{i}, 0\right)\right)=\sum_{i}^{<} x_{i}^{*}(s \varphi)_{\sharp}\left(\alpha_{i}, 0\right) \\
& =\sum_{i}^{<}\left(\sum_{j}^{<}\left(\beta_{j} \varphi_{j i} x_{i}, 0\right)\right)=\sum_{i}^{<}\left(\varphi \alpha_{i} x_{i}, \nabla_{B}\left(\varphi \alpha_{i} x_{i}\right)\right) .
\end{aligned}
$$

Here we have in $G(q, B)$ the equation

$$
\sum_{i}^{<}\left(\varphi \alpha_{i} x_{i}, 0\right)=\left(\varphi x, \sum_{i<t} \Delta_{B}\left(\varphi \alpha_{i} x_{i} \otimes \varphi \alpha_{t} x_{t}\right)\right) .
$$

This yields the result in (3.15).

We now describe a cocycle $\delta$ in the class $\beta_{1} \beta_{2}\left(1_{\Gamma}\right)$. For this let $A, B, C$ be ordered direct sums of cyclic groups and consider homomorphisms

$$
\psi \varphi: A \stackrel{\varphi}{\rightarrow} B \stackrel{\psi}{\rightarrow} C .
$$

Let $r_{A}=r^{<}$be the retraction of $H$ in $(3.10)(3)$ :

$$
\Gamma(A) \otimes \mathbb{Z} / 2 \underset{r_{A}}{\stackrel{H}{\rightleftarrows}} \otimes^{2}(A) \otimes \mathbb{Z} / 2 \quad\left(\text { see } S_{2}(A) \text { in }(3.3)\right) .
$$

Moreover, let $s_{A}$ be a splitting of $\sigma$ :

$$
\Gamma(A) \otimes \mathbb{Z} / 2 \underset{s_{A}}{\stackrel{\sigma}{\rightleftarrows}} A \otimes \mathbb{Z} / 2 \quad\left(\text { see } S_{1}(A) \text { in }(3.3)\right)
$$

defined by

$$
s_{A}\left(\sum_{i} x_{i} \alpha_{i} \otimes 1\right)=\sum_{i} x_{i} \gamma\left(\alpha_{i}\right) \otimes 1 .
$$

Here the $\alpha_{i}$ are the generators of $A$ as in (3.7). We now obtain derivations $D_{1}, D_{2}$ by setting

$$
D_{2}(\psi) q=-\psi_{*} r_{B}+\psi^{*} r_{C}, \quad P D_{1}(\varphi)=-\varphi_{*} s_{A}+\varphi^{*} s_{B} .
$$

For this we use the exact sequences $S_{i}(A)$ in (3.3). We define a 2-cocycle $\delta$ which carries $(\psi, \varphi)$ to the composition

$$
\delta(\psi, \varphi): A \otimes \mathbb{Z} / 2 \stackrel{D_{1}(\varphi)}{\longrightarrow} \Lambda^{2}(B) \otimes \mathbb{Z} / 2 \stackrel{D_{2}(\psi)}{\longrightarrow} \Gamma(C) \otimes \mathbb{Z} / 2
$$

and we observe 
(3.17) Lemma. We have

$$
\beta_{1} \beta_{2}\left(1_{\Gamma}\right)=\{\delta\},
$$

where $\beta_{1}, \beta_{2}$ are the Bockstein homomorphisms in (3.4).

We leave the proof of the lemma as an exercise. The lemma yields a cocycle representing the right-hand side in (3.6).

Next we determine the cocycle $\Delta_{s}$ in (3.9). For this we use the injection

$$
g: \operatorname{Ext}(A, \Gamma C) \subset \underset{q>1}{X} \operatorname{Hom}(\operatorname{Hom}(\mathbb{Z} / q, A), \operatorname{Ext}(\mathbb{Z} / q, \Gamma C)) .
$$

The element $g \Delta_{s}(\psi, \varphi)$ is given by the $\mathbb{Z} / q$-natural homomorphism

$$
\left(g \Delta_{s}(\psi, \varphi)\right)_{q}: \operatorname{Hom}(\mathbb{Z} / q, A) \rightarrow \operatorname{Ext}(\mathbb{Z} / q, \Gamma C)
$$

which satisfies

$$
\left(g \Delta_{s}(\psi, \varphi)\right)_{q}(x)=\Gamma(\psi)_{*} \nabla(\varphi)(x)+\nabla(\psi)(\varphi x)-\nabla(\psi \varphi)(x) .
$$

This equation is an easy consequence of (3.14). As in the remark following (3.12) we may assume that $\nabla_{A}=\nabla_{B}=\nabla_{C}=0$ are trivial. Moreover, we may assume that $q$ is even since $\left(g \Delta_{s}(\psi, \varphi)\right)_{q}$ is trivial if $q$ is odd. We define a function

$$
\begin{aligned}
& \varrho_{A}: A \otimes \mathbb{Z} / 2 \rightarrow \Lambda^{2}(A \otimes \mathbb{Z} / 2), \\
& \varrho_{A}\left(\sum_{i} x_{i} \alpha_{i} \otimes 1\right)=\sum_{i<t}\left(x_{i} \alpha_{i} \otimes 1\right) \wedge\left(x_{t} \alpha_{t} \otimes 1\right) .
\end{aligned}
$$

(3.18) Lemma. $\nabla(\varphi)(x)=\chi_{q} D_{2}(\varphi) \varrho_{A}(x \otimes \mathbb{Z} / 2)$.

Here we have $x \in \operatorname{Hom}(\mathbb{Z} / q, A)$ and

$$
x \otimes \mathbb{Z} / 2 \in \operatorname{Hom}(\mathbb{Z} / q \otimes \mathbb{Z} / 2, A \otimes \mathbb{Z} / 2)=A \otimes \mathbb{Z} / 2
$$

since $q$ is even. Moreover, $\chi_{q}$ in Lemma (3.18) is the composition

$$
\chi_{q}: \Gamma(B) \otimes \mathbb{Z} / 2=\operatorname{Ext}(\mathbb{Z} / 2, \Gamma B) \rightarrow \operatorname{Ext}(\mathbb{Z} / q, \Gamma B)
$$

induced by $\mathbb{Z} / q \rightarrow \mathbb{Z} / q \otimes \mathbb{Z} / 2=\mathbb{Z} / 2$. Lemma (3.18) is a consequence of the formula in (3.15) and the definition of $r_{A}=r^{<}$in (3.10)(3). We apply Lemma (3.18) to the formula for $\left(g \Delta_{s}(\psi, \varphi)\right)_{q}$ above and for $\bar{x}=x \otimes \mathbb{Z} / 2$ we get

(3.19) Lemma. $\left(g \Delta_{s}(\psi, \varphi)\right)_{q}(x)=\chi_{q} D_{2}(\psi)\left(\varrho_{B}(\varphi \bar{x})-\varphi_{*} \varrho_{A}(\bar{x})\right)$.

This follows easily from (3.18) since $D_{1}$ is a derivation. Finally, we observe:

(3.20) Lemma. $\varrho_{B}(\varphi \bar{x})-\varphi_{*} \varrho_{A}(\bar{x})=D_{1}(\varphi)(\bar{x})$.

The proof of Lemma (3.20) requires a lengthy computation with the definitions of $\varrho_{B}, \varrho_{A}$ and $D_{2}(\varphi)$. By (3.19) and (3.20) we thus get

$$
\left(g \Delta_{s}(\psi, \varphi)\right)_{q}(x)=\chi_{q} D_{2}(\psi) D_{1}(\varphi)(\bar{x})
$$


and this yields the formula in (3.6). In fact, (3.21) yields an easy algebraic description of the cocycle $\Delta_{s}$ in terms of the derivations $D_{1}$ and $D_{2}$ above since $g$ is injective.

4. On the cohomology class $\{$ nil $\}$ and James-Hopf invariants on $\mathbf{M}^{2}$. In this section we prove a further formula for the class $\left\{\mathbf{M}^{2}\right\}$, which, however, does not determine $\left\{\mathbf{M}^{2}\right\}$ completely.

For the exterior square $\Lambda^{2}(B)$ of an abelian group $B$ we have the exact sequence (3.1) which induces the exact sequence

$$
\operatorname{Ext}(A, \Gamma B) \stackrel{H_{*}}{\rightarrow} \operatorname{Ext}\left(A, \otimes^{2} B\right) \stackrel{q_{*}}{\rightarrow} \operatorname{Ext}\left(A, \Lambda^{2} B\right) \rightarrow 0
$$

and hence we have the binatural short exact sequence

$$
H_{*} \operatorname{Ext}(A, \Gamma B) \stackrel{i}{\longmapsto} \operatorname{Ext}\left(A, \otimes^{2} B\right) \rightarrow \operatorname{Ext}\left(A, \Lambda^{2} B\right)
$$

together with the surjective map

$$
H^{\prime}: \operatorname{Ext}(A, \Gamma B) \rightarrow H_{*} \operatorname{Ext}(A, \Gamma B)
$$

induced by $H_{*}$. The short exact sequence induces the Bockstein homomorphism

$$
\beta: H^{1}\left(\mathbf{A b}, \operatorname{Ext}\left(-, \Lambda^{2}\right)\right) \rightarrow H^{2}\left(\mathbf{A b}, H_{*} \operatorname{Ext}(-, \Gamma)\right) .
$$

(4.2) Theorem. The algebraic class $\{$ nil $\} \in H^{1}\left(\mathbf{A b}, \operatorname{Ext}\left(-, \Lambda^{2}\right)\right)$ defined below and the class $\left\{\mathbf{M}^{2}\right\}$ of the homotopy category of Moore spaces in degree 2 satisfy the formula

$$
H_{*}^{\prime}\left\{\mathbf{M}^{2}\right\}=\beta\{\operatorname{nil}\} \in H^{2}\left(\mathbf{A} \mathbf{b}, H_{*} \operatorname{Ext}(-, \Gamma)\right) .
$$

This result is true in the cohomology of $\mathbf{A b}$. For the algebraic definition of the class $\{$ nil $\}$ we need the following linear extension nil.

(4.3) Definition. Let $\langle Z\rangle$ be the free group generated by the set $Z$ and let $\Gamma_{n}\langle Z\rangle$ be the subgroup generated by $n$-fold commutators. Then

$$
A=\langle Z\rangle / \Gamma_{2}\langle Z\rangle=\bigoplus_{Z} \mathbb{Z}
$$

is the free abelian group generated by $Z$ and

$$
E_{A}=\langle Z\rangle / \Gamma_{3}\langle Z\rangle
$$

is the free nil(2)-group generated by $Z$.

We have the classical central extension of groups

$$
\Lambda^{2} A \stackrel{w}{\longmapsto} E_{A} \stackrel{q}{\rightarrow} A .
$$

The map $w$ is induced by the commutator map with

$$
w(q x \wedge q y)=x^{-1} y^{-1} x y .
$$


Here the right-hand side denotes the commutator in the group $E_{A}$. Using (3) we get the linear extension of categories (compare also [3], [8])

$$
\operatorname{Hom}\left(-, \Lambda^{2}-\right) \stackrel{+}{\longmapsto} \mathbf{n i l} \stackrel{\mathrm{ab}}{\rightarrow} \mathbf{a b} .
$$

Here $\mathbf{a b}$ and nil are the full subcategories of the category of groups consisting of free abelian groups and free nil(2)-groups respectively. The functor $\mathbf{a b}$ in (3) is abelianization and the action + is given by

$$
f+\alpha=f+w \alpha q
$$

for $f: E_{A} \rightarrow E_{B}, \alpha \in \operatorname{Hom}\left(A, \Lambda^{2} B\right)$. The right-hand side of (6) is a well defined homomorphism since (3) is central.

(4.4) Definition. We define a derivation

$$
\text { nil }: \mathbf{A b} \rightarrow \operatorname{Ext}\left(-, \Lambda^{2}\right)
$$

which carries a homomorphism $\varphi: A \rightarrow B$ in $\mathbf{A b}$ to an element nil $(\varphi) \in$ $\operatorname{Ext}\left(A, \Lambda^{2} B\right)$. The cohomology class $\{$ nil $\}$ represented by the derivation nil is the class used in (4.2). For the definition of nil we choose for each abelian group $A$ a short exact sequence

$$
0 \rightarrow A_{1} \stackrel{d_{A}}{\rightarrow} A_{0} \stackrel{q}{\rightarrow} A \rightarrow 0
$$

where $A_{0}, A_{1}$ are free abelian groups. We also choose a homomorphism $\bar{d}_{A}$ : $E_{A_{1}} \rightarrow E_{A_{0}}$ between free nil(2)-groups such that the abelianization of $\bar{d}_{A}$ is $d_{A}$. For the homomorphism $\varphi: A \rightarrow B$ we choose a commutative diagram in $\mathrm{Ab}$

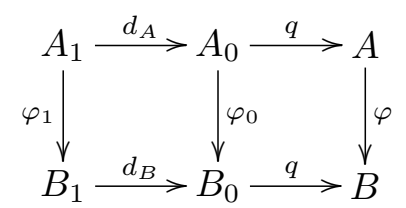

and we choose a diagram of homomorphisms

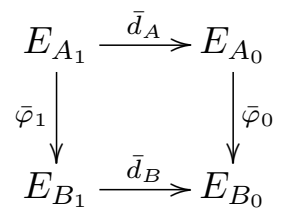

which by abelianization induces $\left(\varphi_{0}, \varphi_{1}\right)$. This diagram, in general, cannot be chosen to be commutative. Since, however, $\varphi_{0} d_{A}=d_{B} \varphi_{1}$ there is a unique element $\alpha \in \operatorname{Hom}\left(A_{1}, \Lambda^{2} B_{0}\right)$ with

$$
\bar{\varphi}_{0} \bar{d}_{A}+\alpha=\bar{d}_{B} \bar{\varphi}_{1}
$$

Here we use the action in $(4.3)(6)$. Now let

$$
\operatorname{nil}(\varphi) \in \operatorname{Ext}\left(A, \Lambda^{2} B\right)=\operatorname{Hom}\left(A_{1}, \Lambda^{2} B\right) / d_{A}^{*} \operatorname{Hom}\left(A_{0}, \Lambda^{2} B\right)
$$


be the element represented by the composition

$$
\left(\Lambda^{2} q\right) \alpha: A_{1} \rightarrow \Lambda^{2} B_{0} \rightarrow \Lambda^{2} B .
$$

One can check that nil $(\varphi)$ does not depend on the choice of $\left(\varphi_{0}, \varphi_{1}\right)$ and $\left(\bar{\varphi}_{0}, \bar{\varphi}_{1}\right)$ and that nil is a derivation, that is, $\operatorname{nil}(\varphi \psi)=\varphi_{*} \operatorname{nil}(\psi)+\psi^{*} \operatorname{nil}(\varphi)$. This completes the definition of the cohomology class $\{$ nil $\}$.

Next we use the derivation $D_{1}$ on $\mathbf{A b}$ defined as in (3.16). The derivation $D_{1}$ carries $\varphi: A \rightarrow B$ to

$$
D_{1}(\varphi) \in \operatorname{Hom}\left(A \otimes \mathbb{Z} / 2, \Lambda^{2}(B) \otimes \mathbb{Z} / 2\right)=\operatorname{Ext}\left(A \otimes \mathbb{Z} / 2, \Lambda^{2} B\right)
$$

and hence represents a cohomology class

$$
\left\{D_{1}\right\} \in H^{1}\left(\mathbf{A b}, \operatorname{Ext}\left(-\otimes \mathbb{Z} / 2, \Lambda^{2}\right)\right) .
$$

Let

$$
p_{2}: \operatorname{Ext}\left(A \otimes \mathbb{Z} / 2, \Lambda^{2} B\right) \rightarrow \operatorname{Ext}\left(A, \Lambda^{2} B\right)
$$

be induced by the projection $A \rightarrow A \otimes \mathbb{Z} / 2$.

(4.5) Proposition. Let $\mathbf{A}$ be the full subcategory of $\mathbf{A b}$ consisting of direct sums of cyclic groups. Then

$$
i_{\mathbf{A}}^{*}\left(p_{2}\right)_{*}\left\{D_{1}\right\}=i_{\mathbf{A}}^{*}\{\text { nil }\}
$$

in $H^{1}\left(\mathbf{A}, \operatorname{Ext}\left(-, \Lambda^{2}\right)\right)$.

We do not know whether this formula also holds if we omit $i_{\mathbf{A}}^{*}$. Proposition (4.5) implies that the formulas in (4.2) and (3.6) are compatible. For the proof of (4.5) we need the following properties of nil(2)-groups. A group $G$ is a nil(2)-group if all triple commutators vanish in $G$. The commutators in $G$ yield the central homomorphism

$$
w: \Lambda^{2}\left(G^{\mathrm{ab}}\right) \rightarrow G
$$

where $G \rightarrow G^{\mathrm{ab}}, x \mapsto\{x\}$, is the abelianization of $G$. We define $w$ by the commutator

$$
w(\{x\} \wedge\{y\})=x^{-1} y^{-1} x y
$$

for $x, y \in G$. Let $M$ be a set and let $f: M \rightarrow G$ be a function such that only finitely many elements $f(m), m \in M$, are nontrivial and let $<, \ll$ be two total orderings on the set $M$. Then we have in $G$ the following formula where we write the nonabelian group structure of $G$ additively, the sums are ordered as indicated.

$$
\sum_{m \in M}^{\ll} f(m)=\sum_{m \in M}^{<} f(m)+w\left(\sum_{\substack{m \ll m^{\prime} \\ m^{\prime}<m}}\{f m\} \wedge\left\{f m^{\prime}\right\}\right) .
$$


For $a \in G$ and $n \in \mathbb{Z}$ let $n a=a+\ldots+a$ be the $n$-fold sum in $G$ in case $n \geq 0$, and let $n a=-|n| a$ for $n<0$. Then one gets in $G$ the formula

$$
n \sum_{m \in M}^{<} f(m)=\sum_{m \in M}^{<} n f(m)-w\left(\left(\begin{array}{l}
n \\
2
\end{array}\right) \sum_{m<m^{\prime}}\{f m\} \wedge\left\{f m^{\prime}\right\}\right),
$$

where $\left(\begin{array}{l}n \\ 2\end{array}\right)=n(n-1) / 2$.

Proof of (4.5). Let $A$ and $B$ be direct sums of cyclic groups and let $\varphi: A \rightarrow B$ be given by $\varphi_{j i} \in \mathbb{Z}$ as in (3.8). Let $A_{0}$ be the free group generated by the set of generators $\left\{\alpha_{i}\right\}$ of $A$ and let $A_{1}$ be the free group generated by $\left\{\alpha_{i}: a_{i} \neq 0\right\}$. Then we choose (see (4.4))

$$
\bar{d}_{A}: E_{A_{1}} \rightarrow E_{A_{0}}, \quad \bar{d}_{A}\left(\alpha_{i}\right)=a_{i} \alpha_{i} .
$$

Similarly we define $\bar{d}_{B}$. Moreover, we define $\bar{\varphi}_{1}$ and $\bar{\varphi}_{0}$ by the ordered sum

$$
\bar{\varphi}_{0}\left(\alpha_{i}\right)=\sum_{j}^{<} \varphi_{j i} \beta_{j} \in E_{B_{0}}, \quad \bar{\varphi}_{1}\left(\alpha_{i}\right)=\sum_{j}^{<}\left(a_{i} \varphi_{j i} / b_{j}\right) \beta_{j} \in E_{B_{1}} .
$$

Hence we get $\alpha$ in (4.4) by the formula (see (4.6))

$$
\begin{aligned}
\bar{d}_{B} \bar{\varphi}_{1}\left(\alpha_{i}\right)-\bar{\varphi}_{0} \bar{d}_{A}\left(\alpha_{i}\right) & =\sum_{j}^{<} a_{i} \varphi_{j i} \beta_{j}-a_{i} \sum_{j}^{<} \varphi_{j i} \beta_{j} \\
& =w\left(\begin{array}{c}
a_{i} \\
2
\end{array}\right) \sum_{j<t}\left\{\varphi_{j i} \beta_{j}\right\} \wedge\left\{\varphi_{t i} \beta_{t}\right\} .
\end{aligned}
$$

Hence $\operatorname{nil}(\varphi) \in \operatorname{Ext}\left(A, \Lambda^{2} B\right)$ is given by the formula $\left(\alpha_{i}: \mathbb{Z} / a_{i} \subset A\right.$ as in (3.7))

$$
\left(\alpha_{i}\right)^{*} \operatorname{nil}(\varphi)=\left(\begin{array}{c}
a_{i} \\
2
\end{array}\right) \sum_{j<t} \varphi_{j i} \varphi_{t i}\left(1 \otimes \beta_{j} \wedge \beta_{t}\right),
$$

where $1 \otimes \beta_{j} \wedge \beta_{t} \in \mathbb{Z} / a_{i} \otimes \Lambda^{2} B=\operatorname{Ext}\left(\mathbb{Z} / a_{i}, \Lambda^{2} B\right)$. Using the definition of $D_{1}$ in the proof of (3.16) it is easy to check that $\left(\alpha_{i}\right)^{*} p_{2} D_{1}(\varphi)$ coincides with the right-hand side of the formula so that we actually have $\operatorname{nil}(\varphi)=p_{2} D_{1}(\varphi)$. This proves (4.5).

We will need the following element which projects to nil $(\varphi)$ above.

(4.7) Definition. For $\varphi$ in the proof above let $\overline{\operatorname{nil}}(\varphi) \in \operatorname{Ext}\left(A, \otimes^{2} B\right)$ be given by the formula

$$
\left(\alpha_{2}\right) * \overline{\operatorname{nil}}(\varphi)=\left(\begin{array}{c}
a_{i} \\
2
\end{array}\right) \sum_{j<t} \varphi_{j i} \varphi_{t i}\left(1 \otimes \beta_{j} \otimes \beta_{t}\right) .
$$

We clearly have $\operatorname{Ext}(A, p) \overline{\operatorname{nil}}(\varphi)=\operatorname{nil}(\varphi)$ where $p: \otimes^{2} B \rightarrow \Lambda^{2} B$ is the projection. 
Recall that for the bifunctor $\operatorname{Ext}\left(-, \otimes^{2}\right)$ on $\mathbf{A b}$ we have the canonical split linear extension

$$
\operatorname{Ext}\left(-, \otimes^{2}\right) \longmapsto \mathbf{A b} \times \operatorname{Ext}\left(-, \otimes^{2}\right) \rightarrow \mathbf{A b} .
$$

Objects in $\mathbf{A b} \times \operatorname{Ext}\left(-, \otimes^{2}\right)$ are abelian groups and morphisms $(\varphi, \alpha)$ : $A \rightarrow B$ are given by $\varphi \in \operatorname{Hom}(A, B)$ and $\alpha \in \operatorname{Ext}\left(A, \otimes^{2} B\right)$ with composition $(\varphi, \alpha)(\psi, \beta)=\left(\varphi \psi, \varphi_{*} \beta+\psi^{*} \alpha\right)$. The derivation nil in (4.4) defines a subcategory

$$
\mathbf{A b}(\text { nil }) \subset \mathbf{A b} \times \operatorname{Ext}\left(-, \otimes^{2}\right)
$$

consisting of all morphisms $(\varphi, \alpha): A \rightarrow B$ which satisfy the condition

$$
p_{*}(\alpha)=\operatorname{nil}(\varphi) \in \operatorname{Ext}\left(A, \Lambda^{2} B\right) .
$$

Here $p: \otimes^{2} B \rightarrow \Lambda^{2} B$ induces $p_{*}=\operatorname{Ext}(A, p)$. The exact sequence (4.1) shows that we have a commutative diagram of linear extensions of categories

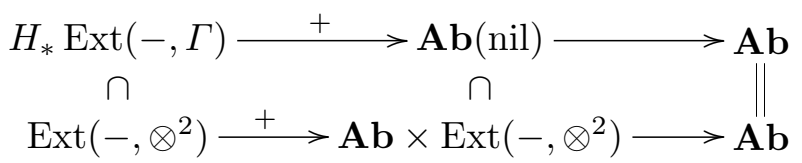

(4.9) Lemma. The cohomology class represented by the linear extension for $\mathbf{A b}$ (nil) satisfies

$$
\{\mathbf{A b}(\text { nil })\}=\beta\{\text { nil }\} \in H^{2}\left(\mathbf{A b}, H_{*} \operatorname{Ext}(-, \Gamma)\right),
$$

where $\beta$ is the Bockstein operator in (4.2).

Proof. Let $s: \operatorname{Ext}\left(A, \Lambda^{2} B\right) \rightarrow \operatorname{Ext}\left(A, \otimes^{2} B\right)$ be a set-theoretic splitting of $\operatorname{Ext}(A, p)=p_{*}$. Then $\beta\{$ nil $\}$ is represented by the 2-cocycle $c=$ $i^{-1} \delta(s$ nil), where $i$ is the inclusion in (4.1) and where $\delta$ is the coboundary in (1.5). Hence $c$ carries the 2-simplex $(\psi, \varphi)$ in $\mathbf{A b}$ to

$$
c(\psi, \varphi)=i^{-1}\left(\psi_{*} s \operatorname{nil}(\varphi)-s \operatorname{nil}(\psi \varphi)+\varphi^{*} s \operatorname{nil}(\psi)\right) .
$$

On the other hand, we define a set-theoretic section $t$ for the linear extension $\mathbf{A b}\left(\right.$ nil) by $t(\varphi)=(\varphi, s \operatorname{nil}(\varphi))$. Then $\Delta_{t}$ in (1.4) is given by

$$
s \operatorname{nil}(\psi \varphi)=\psi_{*} s \operatorname{nil}(\varphi)+\varphi^{*} s \operatorname{nil}(\psi)+i \Delta_{t}(\psi, \varphi) .
$$

Hence $c=-\Delta_{t}$ yields the lemma. In fact, since the elements in (4.9) are of order 2 we can omit the sign.

For Moore spaces $M(A, 2)=\Sigma M_{A}$ and $M(B, 2)=\Sigma M_{B}$ as in (2.2) we have the James-Hopf invariant ([12], [5])

$$
\left[\Sigma M_{A}, \Sigma M_{B}\right] \stackrel{\gamma_{2}}{\rightarrow}\left[\Sigma M_{A}, \Sigma M_{B} \wedge M_{B}\right]=\operatorname{Ext}(A, B \otimes B),
$$

which satisfies for $\alpha \in \operatorname{Ext}(A, \Gamma B)$ the formula

$$
\lambda_{2}(\xi+\alpha)=\lambda_{2}(\xi)+H_{*} \alpha .
$$


Hence $\gamma_{2}$ induces a well defined function

$$
\bar{\gamma}_{2}: \operatorname{Hom}(A, B) \rightarrow \operatorname{Ext}\left(A, \Lambda^{2} B\right)
$$

defined by $\bar{\gamma}_{2}(\varphi)=q_{*} \gamma_{2}(\xi)$ where $\xi$ induces $H_{2}(\xi)=\varphi: A \rightarrow B$. One can check that $\bar{\gamma}_{2}$ is a derivation which represents a cohomology class in $H^{1}\left(\mathbf{A} \mathbf{b}, \operatorname{Ext}\left(-, \Lambda^{2} B\right)\right)$. This cohomology class does not depend on the choice of $M_{A}, M_{B}$ above.

(4.11) Theorem. The cohomology class $\left\{\bar{\gamma}_{2}\right\}$ given by the James-Hopf invariant $\gamma_{2}$ coincides with

$$
\left\{\bar{\gamma}_{2}\right\}=\{\operatorname{nil}\} \in H^{1}\left(\mathbf{A b}, \operatorname{Ext}\left(-, \Lambda^{2}\right)\right) .
$$

Moreover, there is a full functor $\tau$,

$$
\mathbf{M}^{2} \stackrel{\tau}{\rightarrow} \mathbf{A b}(\text { nil }) \stackrel{i}{\subset} \mathbf{A b} \times \operatorname{Ext}\left(-, \otimes^{2}\right),
$$

which is the identity on objects and which is defined on morphisms by

$$
\tau(\xi)=\left(H_{2} \xi, \gamma_{2} \xi\right) .
$$

The functor $\tau$ is part of the following commutative diagram of linear extensions:

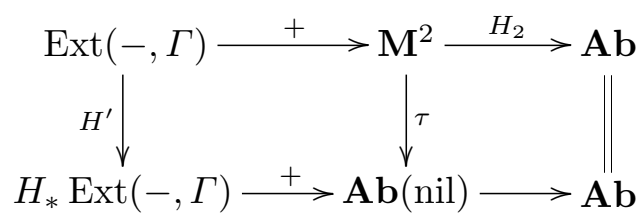

Proof of (4.2). The existence of the functor $\tau$ shows that $H_{*}^{\prime}\left\{\mathbf{M}^{2}\right\}=$ $\{\mathbf{A b}($ nil) $\}$. Therefore we obtain (4.2) by (4.9).

(4.12) R e mark. We can give an alternative description of the functor $\tau$ in (4.11) by use of the singular chain complex of a loop space which yields the Adams-Hilton functor

$$
C_{*} \Omega: \mathrm{Ho}\left(\mathbf{T o p}^{*}\right) \rightarrow \mathrm{Ho}(\mathbf{D A})
$$

between homotopy categories (compare [1] and also [2]). Here DA is the category of $\mathbb{Z}$-chain algebras. The functor $C_{*} \Omega$ restricted to $\mathbf{M}^{2}$ leads to the following diagram where $\widetilde{\mathbf{M}}^{2} \subset \mathrm{Ho}(\mathbf{D A})$ is the full subcategory consisting of $C_{*} \Omega M(A, 2), A \in \mathbf{A b}$ :

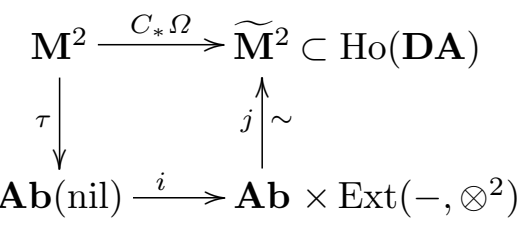


where $j$ is an equivalence of categories such that $j i \tau$ is naturally isomorphic to $C_{*} \Omega$.

Proof of (4.11). The image category of the functor

$$
\tau: \mathbf{M}^{2} \rightarrow \mathbf{A b} \times \operatorname{Ext}\left(-, \otimes^{2}\right)
$$

is $\mathbf{A b}$ (nil) since we show

$$
\bar{\gamma}_{2}=\text { nil }
$$

for compatible choices of $\bar{d}_{A}, d_{A}^{\prime}$ in (4.4) and (2.2). We use the equivalence of linear track extension described in (VI.4.7) of [3]. This shows that a triple $\left(\bar{\varphi}_{0}, \bar{\varphi}_{1}, G\right)$ with $G \in \operatorname{Hom}\left(A_{1}, \otimes^{2} B_{0}\right)$ satisfying $p_{*} G=\alpha$ (see (4.4)) corresponds to a diagram

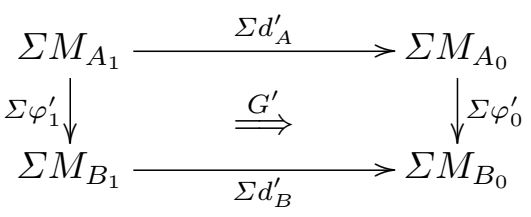

Here $d_{A}^{\prime}$ and $d_{B}^{\prime}$ induce $\bar{d}_{A}$ and $\bar{d}_{B}$ respectively and $\varphi_{0}^{\prime}, \varphi_{1}^{\prime}$ induces $\bar{\varphi}_{0}, \bar{\varphi}_{1}$ in (4.4). The track $G^{\prime}$ is determined by $G$. This track determines a principal map $\bar{\varphi} \in\left[\Sigma M_{A}, \Sigma M_{B}\right]$ such that $\tau(\bar{\varphi})=\left(\varphi,\left(\otimes^{2} q\right)_{*}\{G\}\right)$, where $\{G\} \in$ $\operatorname{Ext}\left(A, \otimes^{2} B_{0}\right)$ is represented by $G$. This follows from the bijection (6)-(11) in (VI.4.7) of [3]. Since $p_{*} G=\alpha$ we get $\bar{\gamma}_{2}=$ nil.

(4.13) ExAmple. Let $A$ and $B$ be direct sums of cyclic groups as in (3.8) and let $s \varphi \in[M(A, 2), M(B, 2)]$ be defined as in (3.9). Then the functor $\tau$ in (4.11) satisfies

$$
\tau(s \varphi)=(\varphi, \overline{\operatorname{nil}}(\varphi)),
$$

where $\overline{\operatorname{nil}}(\varphi)$ is defined in (4.7). We obtain this formula by the methods in the proof of (4.11) above. In this case we can also compute the James-Hopf invariant $\gamma_{2}(s \varphi)$, which actually is $\gamma_{2}(s \varphi)=\overline{\operatorname{nil}}(\varphi)$.

As a corollary of (4.2) we get:

(4.14) Proposition. $\left\{\mathbf{M}^{2}\right\}$ is a (nontrivial) element of order 2.

Proof. We know that multiplication by 2 on $\Gamma(A)$ is the composition

$$
2=P H: \Gamma A \rightarrow \otimes^{2} A \rightarrow \Gamma A,
$$

where $P=[1,1]$. Hence also the composition

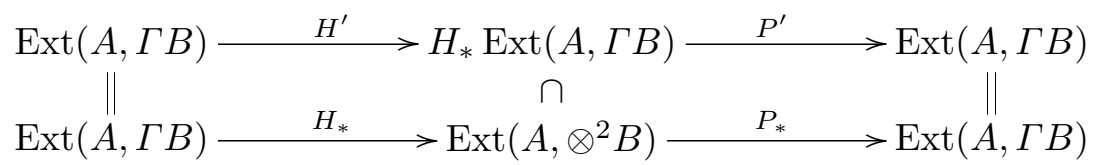


is a multiplication by 2 . Therefore by (4.2) we get

$$
2\left\{\mathbf{M}^{2}\right\}=\left(P^{\prime} H^{\prime}\right)_{*}\left\{\mathbf{M}^{2}\right\}=P_{*}^{\prime} H_{*}^{\prime}\left\{\mathbf{M}^{2}\right\}=P_{*}^{\prime} \beta\{\text { nil }\} .
$$

Here the commutative diagram of short exact sequences

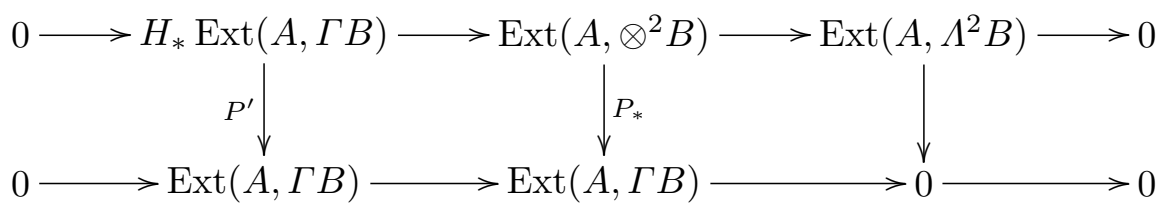

shows that $P_{*}^{\prime} \beta=0$.

(4.15) Proposition. Each element in $H^{1}\left(\mathbf{A b}, \operatorname{Ext}\left(-, \Lambda^{2}\right)\right)$ is of order 2 , in particular, $2\{$ nil $\}=0$.

Proof. Let $A, B$ be abelian groups and let $\varphi \in \operatorname{Hom}(A, B)$. Let $2_{A}=$ 2 id $\in \operatorname{Hom}(A, A)$ be multiplication by 2 . Then we have

$$
\varphi \circ 2_{A}=2 \varphi=2_{B} \circ \varphi \text {. }
$$

Now the derivation property of $N$ with $\{N\} \in H^{1}\left(\mathbf{A b}, \operatorname{Ext}\left(-, \Lambda^{2}\right)\right)$ shows

$$
\begin{aligned}
& N\left(\varphi \circ 2_{A}\right)=\varphi_{*} N\left(2_{A}\right)+\left(2_{A}\right)^{*} N(\varphi)=\varphi_{*} N\left(2_{A}\right)+2 N(\varphi), \\
& N\left(2_{B} \circ \varphi\right)=\left(2_{B}\right)_{*} N(\varphi)+\varphi^{*} N\left(2_{B}\right)=4 N(\varphi)+\varphi^{*} N\left(2_{B}\right) .
\end{aligned}
$$

Hence we get $2 N(\varphi)=\varphi_{*} N\left(2_{A}\right)-\varphi^{*} N\left(2_{B}\right)$, so that $2 N$ is an inner derivation.

5. A subcategory of $\mathbf{M}^{2}$ given by diagonal elements. Let $\mathbb{Z} / 2 * A$ be the 2-torsion of the abelian group $A$. We construct a subcategory $\mathbf{H}$ of the category of Moore spaces $\mathbf{M}^{2}$ with the following property.

(5.1) TheOREM. There exists a subcategory $\mathbf{H}$ of $\mathbf{M}^{2}$ together with a commutative diagram of linear extensions

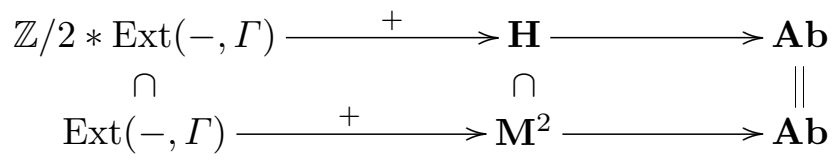

The theorem shows that the class $\left\{\mathbf{M}^{2}\right\}$ is in the image of

$$
i_{*}: H^{2}(\mathbf{A b}, \mathbb{Z} / 2 * \operatorname{Ext}(-, \Gamma)) \rightarrow H^{2}(\mathbf{A b}, \operatorname{Ext}(-, \Gamma)),
$$

where $i$ is the inclusion $\mathbb{Z} / 2 * \operatorname{Ext}(A, \Gamma(B)) \subset \operatorname{Ext}(A, \Gamma(B))$.

(5.2) Corollary. The extension $\mathbf{M}^{2} \rightarrow \mathbf{A b}$ is split on any full subcategory of $\mathbf{A b}$ consisting of objects $A, B$ with $(\mathbb{Z} / 2) * \operatorname{Ext}(A, \Gamma B)=0$.

(5.3) Corollary. Let $A$ be any abelian group for which the 2-torsion of $\operatorname{Ext}(A, \Gamma A)$ is trivial. Then the group of homotopy equivalences of $M(A, 2)$ 
is given by the split extension

$$
\operatorname{Ext}(A, \Gamma A) \longmapsto \mathfrak{E}(M(A, 2)) \rightarrow \operatorname{Aut}(A),
$$

where $\varphi \in \operatorname{Aut}(A)$ acts on $a \in \operatorname{Ext}(A, \Gamma A)$ by $\varphi \cdot a=(\Gamma \varphi)_{*}\left(\varphi^{-1}\right)^{*}(a)$.

Proof of (5.1). For a Moore space $M(A, 2)=\Sigma M_{A}$ we have the diagonal element

$$
\Delta_{A} \in\left[\Sigma M_{A}, \Sigma M_{A} \wedge M_{A}\right]=\operatorname{Ext}(A, A \otimes A)
$$

which is given by the suspension of the reduced diagonal $M_{A} \rightarrow M_{A} \wedge M_{A}$. Let $\left[1_{A}, 1_{A}\right]: \Sigma M_{A} \wedge M_{A} \rightarrow \Sigma M_{A}$ be the Whitehead product for the identity $1_{A}$ of $\Sigma M_{A}$. Then

$$
\left[1_{A}, 1_{A}\right] \Delta_{A}=-1_{A}-1_{A}+1_{A}+1_{A}=0
$$

is the trivial commutator. This implies that also

$$
\Delta_{A} \in \operatorname{Ker}\left\{[1,1]_{*}: \operatorname{Ext}(A, A \otimes A) \rightarrow \operatorname{Ext}(A, \Gamma A)\right\}
$$

with $[1,1]$ in (3.2). We have the short exact sequences (see (3.3))

$$
0 \rightarrow \operatorname{Ext}(A, \Gamma(A) \otimes \mathbb{Z} / 2) \stackrel{H_{*}}{\longrightarrow} \operatorname{Ext}\left(A, \otimes^{2}(A) \otimes \mathbb{Z} / 2\right) \stackrel{q_{*}}{\longrightarrow} \operatorname{Ext}\left(A, \Lambda^{2}(A) \otimes \mathbb{Z} / 2\right) \rightarrow 0
$$

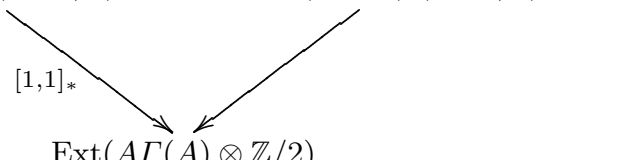

which shows by (3) that for the projection $p: \otimes^{2} A \rightarrow\left(\otimes^{2} A\right) \otimes \mathbb{Z} / 2$ there is a unique element $\Delta_{A}^{\prime} \in \operatorname{Ext}(A, \Gamma(A) \otimes \mathbb{Z} / 2)$ with

$$
H_{*} \Delta_{A}^{\prime}=p_{*} \Delta_{A} .
$$

Now, using the surjection $p_{*}: \operatorname{Ext}(A, \Gamma A) \rightarrow \operatorname{Ext}(A, \Gamma(A) \otimes \mathbb{Z} / 2)$, we choose an element $\Delta_{A}^{\prime \prime} \in \operatorname{Ext}(A, \Gamma A)$ with

$$
p_{*} \Delta_{A}^{\prime \prime}=\Delta_{A}^{\prime} \text {. }
$$

We call $\Delta_{A}^{\prime \prime}$ a diagonal structure for $A$. For the definition of the subcategory $\mathbf{H}$ in $\mathbf{M}^{2}$ we choose such a diagonal structure for each abelian group $A$ in Ab. We define the set of morphisms in $\mathbf{H}$ with

$$
\mathbf{H}(A, B) \subset\left[\Sigma M_{A}, \Sigma M_{B}\right]
$$

by the composition (compare (4.10))

$$
\left[\Sigma M_{A}, \Sigma M_{B}\right] \stackrel{\gamma_{2}}{\longrightarrow} \operatorname{Ext}(A, B \otimes B) \stackrel{[1,1]_{*}}{\longrightarrow} \operatorname{Ext}(A, \Gamma B),
$$

and by the diagonal structures $\Delta_{A}^{\prime \prime}, \Delta_{B}^{\prime \prime}$, namely

$$
\bar{\varphi} \in \mathbf{H}(A, B) \Leftrightarrow[1,1]_{*} \gamma_{2} \bar{\varphi}=-\varphi_{*} \Delta_{A}^{\prime \prime}+\varphi^{*} \Delta_{B}^{\prime \prime} .
$$

We show that for $\bar{\varphi} \in \mathbf{H}(A, B)$ and $\bar{\psi} \in \mathbf{H}(B, C)$ we actually have $\bar{\psi} \bar{\varphi} \in \mathbf{H}(A, C)$ so that $\mathbf{H}$ is a well defined subcategory of $\mathbf{M}^{2}$. For this we 
need the fact that $\gamma_{2}$ is a derivation, that is,

$$
\gamma_{2}(\bar{\psi} \bar{\varphi})=\psi_{*} \gamma_{2}(\bar{\varphi})+\varphi^{*} \gamma_{2}(\bar{\varphi})
$$

Hence we get

$$
\begin{aligned}
{[1,1]_{*} \gamma_{2}(\bar{\psi} \bar{\varphi}) } & =[1,1]_{*}\left(\psi_{*} \gamma_{2}(\bar{\varphi})+\varphi^{*} \gamma_{2}(\bar{\psi})\right) \\
& =\psi_{*}[1,1]_{*} \gamma_{2}(\bar{\varphi})+\varphi^{*}[1,1]_{*} \gamma_{2}(\bar{\varphi}) \\
& =\psi_{*}\left(-\varphi_{*} \Delta_{A}^{\prime \prime}+\varphi^{*} \Delta_{B}^{\prime \prime}\right)+\varphi^{*}\left(-\psi_{*} \Delta_{B}^{\prime \prime}+\psi^{*} \Delta_{C}^{\prime \prime}\right) \\
& =-(\psi \varphi)_{*} \Delta_{A}^{\prime \prime}+(\psi \varphi)^{*} \Delta_{C}^{\prime \prime} .
\end{aligned}
$$

The crucial observation needed for the proof of Theorem (5.1) is the following equation where we use the interchange map $T: B \otimes B \rightarrow B \otimes B$ with $T(x \otimes y)=y \otimes x:$

$$
(1-T)_{*} \gamma_{2}(\bar{\varphi})=\varphi_{*} \Delta_{A}-\varphi^{*} \Delta_{B}
$$

This equation follows from the corresponding known property of JamesHopf invariants (Appendix A of [4]) with respect to "cup products" which in our case has the form

$$
\bar{\varphi} \cup \bar{\varphi}=\Delta_{1,1} \bar{\varphi}+\left(1+T_{2,1}\right) \gamma_{2}(\bar{\varphi}) .
$$

This equation is equivalent to (8). We now consider the following commutative diagram.

$$
\begin{array}{ccccc}
\operatorname{Ext}(A, \Gamma B) & = & \operatorname{Ext}(A, \Gamma B) & = & \operatorname{Ext}(A, \Gamma B) \\
\downarrow+ & & \downarrow H_{*} & & \downarrow \cdot 2 \\
{\left[\Sigma M_{A}, \Sigma M_{B}\right]} & \stackrel{\gamma_{2}}{\longrightarrow} & \operatorname{Ext}(A, B \otimes B) & \stackrel{[1,1]_{*}}{\longrightarrow} & \operatorname{Ext}(A, \Gamma B) \\
\downarrow^{\mu} & & \downarrow & & \downarrow \\
\operatorname{Hom}(A, B) & \stackrel{\bar{\gamma}_{2}}{\longrightarrow} & \operatorname{Ext}\left(A, \Lambda^{2} B\right) & \stackrel{[1,1]_{*}}{\longrightarrow} & \operatorname{Ext}(A, \Gamma(B) \otimes \mathbb{Z} / 2)
\end{array}
$$

The columns are exact sequences. Here $\gamma_{2}$ is not a homomorphism; since, however, (4.10)(1) holds we see that the induced function $\bar{\gamma}_{2}$ is well defined. Moreover, we use $[1,1] H=\cdot 2$ so that $[1,1]_{*}$ in the bottom row is well defined.

We now claim that (8) implies the formula

$$
[1,1]_{*} \bar{\gamma}_{2}(\varphi)=-\varphi_{*} \Delta_{A}^{\prime}+\varphi^{*} \Delta_{B}^{\prime}
$$

By the diagram above this shows that for any $\varphi \in \operatorname{Hom}(A, B)$ there is an element $\bar{\varphi}$ which satisfies the condition in (7). Thus the functor $\underline{\underline{H}} \rightarrow \mathbf{A b}$ is full, moreover the diagram above shows that $\mathbf{H}$ is part of a linear extension as described in the theorem. In fact, for $\bar{\varphi} \in \mathbf{H}(A, B)$ we have $\bar{\varphi}+\alpha \in \mathbf{H}(A, B)$ if and only if $2 \alpha=0$. 
It remains to prove (9). For this consider the commutative diagram

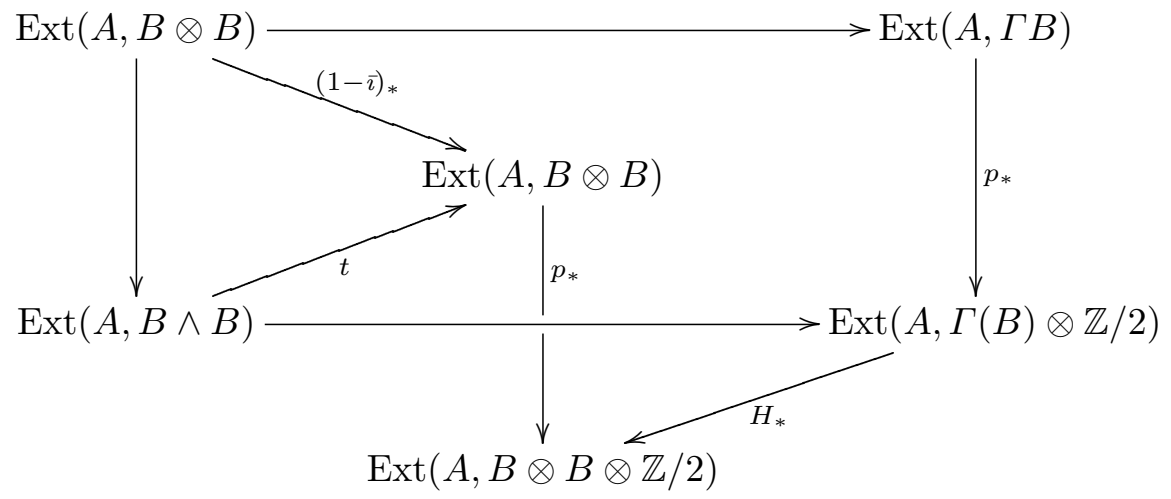

The square in this diagram coincides with the corresponding square in the diagram above. Since for $x \otimes y \in B \otimes B$,

$$
H[1,1](x \otimes y)=x \otimes y+y \otimes x \equiv x \otimes y-y \otimes x \bmod 2,
$$

we see that the diagram commutes. The homomorphism $t$ is induced by $1-T$. On the other hand, $H_{*}$ in the diagram is injective. This shows that (9) holds by the following equations:

$$
\begin{aligned}
H_{*}[1,1]_{*} \bar{\gamma}_{2}(\varphi) & =H_{*} p_{*}[1,1]_{*} \gamma_{2} \bar{\varphi}=p_{*}(1-T)_{*} \gamma_{2} \bar{\varphi} \\
& =p_{*}\left(\varphi_{*} \Delta_{A}-\varphi^{*} \Delta_{B}\right)=\varphi_{*}\left(p_{*} \Delta_{A}\right)-\varphi^{*}\left(p_{*} \Delta_{B}\right) \\
& =\varphi_{*}\left(H_{*} \Delta_{A}^{\prime}\right)-\varphi^{*}\left(H_{*} \Delta_{B}^{\prime}\right)=H_{*}\left(\varphi_{*} \Delta_{A}^{\prime}-\varphi^{*} \Delta_{B}^{\prime}\right) .
\end{aligned}
$$

This completes the proof of Theorem (5.1).

Formula (9) in the proof of (5.1) above and (1) in the proof of (4.11) show

$$
[1,1]_{*} \operatorname{nil}(\varphi)=[1,1]_{*} \bar{\varphi}_{2}(\varphi)=-\varphi_{*} \Delta_{A}^{\prime}+\varphi^{*} \Delta_{B}^{\prime}
$$

Hence the composition $[1,1]_{*}$ nil with

$$
[1,1]_{*}: \operatorname{Ext}\left(A, \Lambda^{2} B\right) \rightarrow \operatorname{Ext}(A, \Gamma B \otimes \mathbb{Z} / 2)
$$

is an inner derivation. This implies

(5.4) Proposition. We have

$$
[1,1]_{*}\{\text { nil }\}=0
$$

in $H^{1}(\mathbf{A} \mathbf{b}, \operatorname{Ext}(-, \mathbb{Z} / 2 \otimes \Gamma))$.

\section{References}

[1] J. F. Adams and P. J. Hilton, On the chain algebra of a loop space, Comment. Math. Helv. 30 (1956), 305-330. 
[2] H.-J. Baues, Algebraic Homotopy, Cambridge Stud. Adv. Math. 15, Cambridge University Press, 1988.

[3] - Combinatorial Homotopy and 4-Dimensional Complexes, de Gruyter, Berlin, 1991.

[4] - Homotopy Type and Homology, Oxford Math. Monograph, Oxford University Press, 1996.

[5] - Commutator Calculus and Groups of Homotopy Classes, London Math. Soc. Lecture Note Ser. 50, Cambridge University Press, 1981.

[6] -, Homotopy types, in: Handbook of Algebraic Topology, Chapter I, I. M. James (ed.), Elsevier, 1995, 1-72.

[7] - On the cohomology of categories, universal Toda brackets, and homotopy pairs, $K$-Theory, to appear.

[8] H.-J. Baues and W. Dreckmann, The cohomology of homotopy categories and the general linear group, ibid. 3 (1989), 307-338.

[9] H.-J. Baues and G. Wirsching, The cohomology of small categories, J. Pure Appl. Algebra 38 (1985), 187-211.

[10] K. A. Hardie, On the category of homotopy pairs, Topology Appl. 14 (1982), 59-69.

[11] P. Hilton, Homotopy Theory and Duality, Gordon and Breach, 1965.

[12] I. M. James, Reduced product spaces, Ann. of Math. 62 (1955), 170-197.

[13] M. Jibladze and T. Pir ashvili, Cohomology of algebraic theories, J. Algebra 137 (1991), 253-296.

[14] T. Pirashvili and F. Waldhausen, MacLane homology and topological Hochschild homology, J. Pure Appl. Algebra 82 (1992), 81-98.

[15] J. H. C. Whitehead, A certain exact sequence, Ann. of Math. 52 (1950), 51-110.

Max-Planck-Institut für Mathematik

Gottfried-Claren-Str. 26

D-53225 Bonn, Germany

E-mail: baues@mpim-bonn.mpg.de
Département de Mathématiques Université de Valenciennes B.P. 311 59304 Valenciennes, France E-mail: hartl@univ-valenciennes.fr 